\title{
7 AJUSTAMENTO EMOCIONAL EM DOENTES COM SARCOMA E A PERCEÇÃO DE SUPORTE AUTÓNOMO DADO PELOS ENFERMEIROS
}

\author{
| Celeste Bastos ${ }^{1}$; Célia Santos²; Jorge Freitas³ Emilia Magalhães' Lígia Lima ${ }^{5} \mid$
}

\section{RESUMO}

Os sarcomas são tumores malignos raros, com consequências negativas para a funcionalidade e autoimagem, e que se associam a um maior risco de perturbação emocional, revelando-se fundamental estudar o papel dos enfermeiros no suporte fornecido a estes doentes.

O objetivo do estudo foi analisar a relação entre o ajustamento emocional e a perceção sobre o ambiente terapêutico, em três momentos do processo de tratamento.

Realizou-se um estudo longitudinal, numa amostra de 24 pessoas com sarcoma (ósseo ou dos tecidos moles), com uma média de 41 anos de idade e de 10 anos de escolaridade. Os instrumentos utilizados foram: questionário de caracterização sociodemográfica e clínica, a versão portuguesa da Escala de Ansiedade e Depressão Hospitalar (EADH) e o Questionário de Perceção do Cliente sobre o Ambiente Terapêutico (QPCAT).

Globalmente foram identificados níveis baixos de ansiedade e de depressão ao longo do tratamento. Os doentes demonstraram uma perceção favorável sobre o suporte proporcionado pelos enfermeiros, com repercussões positivas no seu ajustamento emocional, em especial nas fases mais avançadas do tratamento.

Os resultados sugerem que os enfermeiros apoiam e encorajam os doentes na gestão do seu tratamento, sendo este suporte promotor de um bom ajustamento psicossocial. São ainda evidenciados alguns indicadores de maior vulnerabilidade que requerem um suporte terapêutico ajustado às suas necessidades ao longo do percurso da doença e do tratamento.

PALAVRAS-CHAVE: Sarcoma; Ansiedade; Depressão; Autonomia pessoal

\section{RESUMEN}

\section{“Ajuste emocional de los pacientes con sarcoma y percepción de apoyo autonomo de los enfermeros"}

Los sarcomas son tumores malignos raros, con consecuencias negativas para la funcionalidad y la propia imagen. Los sarcomas se asocian a un mayor riesgo de Trastorno emocional, e es indispensable estudiar el papel de enfermería en el apoyo a estos pacientes. El objetivo de este estudio fue analizar la relación entre ajuste emocional y la percepción de autonomía en el ambiente terapéutico, en dos momentos del tratamiento. Se realizó un estudio longitudinal en una muestra de 24 personas con sarcoma de tejidos blandos o hueso, con una media de 41 años de edad y 10 años de escolaridad. Los instrumentos utilizados fueron: un cuestionario encuesta sociodemográfico y caracterización clínica, la versión portuguesa de la escala de ansiedad e depresión en el hospital (HADS) e la escala sobre el medio ambiente terapéutico (QPCAT). Los pacientes demostraron un apoyo favorable de los enfermeros, con repercusiones positivas en su ajuste emocional, particularmente en las etapas posteriores del tratamiento. Los resultados sugieren que las enfermeras apoyan y animan los pacientes en el manejo de su tratamiento, que es un buen promotor del ajuste psicosocial. Aún se evidencian algunos indicadores de mayor vulnerabilidad que requieren de un apoyo terapéutico ajustado a sus necesidades a través del curso de la enfermedad y el tratamiento.

\section{DESCRIPTORES: Sarcoma; Ansiedad; Depresión; Autonomía} personal

\section{ABSTRACT \\ "Emotional adjustment in sarcoma patients and perceived au- tonomy support provided by nurses"}

Sarcomas are a rare type of solid cancer associated with several negative psychological outcomes in terms of functionality and self-image. There is an increased risk of emotional distress in sarcoma patients therefore it's important to study how nurses can assist these patients. This is a longitudinal study aimed to study the association between emotional adjustment of patients with sarcoma and the perceived autonomy supportiveness provided by nurses Participants were 24 adult patients of both sexes, mean age of $41(\mathrm{SD}=16,8)$, diagnosed with sarcoma. The instruments used were: Sociodemographic and Clinical Questionnaire,

the Health Care Climate Questionnaire (HCCQ) and the Hospital Anxiety and Depression Scale (HADS).

Overall, low levels of emotional distress were found during treatment. Patients reported a positive perception of perceived autonomy support provided by nurses, which proved to be associated with lower emotional distress, especially during the advanced phases of treatment. The study emphasizes the importance of the autonomous support provided by nurses in the disease management process, and gives clues to the identification of the most vulnerable patients in terms of emotional distress, in order to plan psychosocial interventions to promote patients' wellbeing.

\section{KEYWORDS: Sarcoma; Anxiety; Depression; Personal au- tonomy}

Submetido em 03-11-2015

Aceite em 30-05-2016

1 Doutora em Psicologia; Professora Adjunta na Escola Superior de Enfermagem do Porto, Rua Dr. António Bernardino de Almeida, Porto, Portugal, cbastos@esenf.pt

2 Doutora em Psicologia; Professora Coordenadora na Escola Superior de Enfermagem do Porto, Porto, Portugal, celiasantos@esenf.pt

3 Mestre em Bioética; Enfermeiro Especialista no Instituto Português de Oncologia do Porto, Porto, Portugal, mjorgefreitas@sapo.pt

4 Curso de Estudos Superiores Especializados em Enfermagem de Saúde Mental; Enfermeira no Instituto Português de Oncologia do Porto, Porto, Portugal.

5 Doutora em Psicologia; Professora Coordenadora na Escola Superior de Enfermagem do Porto, Porto, Portugal, ligia@esenf.pt

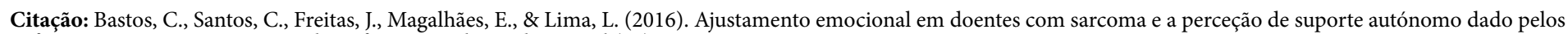
enfermeiros. Revista Portuguesa de Enfermagem de Saúde Mental (16), 49-58. 


\section{INTRODUÇÃO}

Os sarcomas são um tipo raro de cancro sólido, que afeta o tecido conjuntivo ou o tecido ósseo. Os sarcomas correspondem a mais de $20 \%$ de todos os cancros sólidos pediátricos e menos de $1 \%$ no caso do adulto (Burningham, HashibE, Spector, \& Schiffman, 2012). Embora relativamente raro, este tipo de cancro é frequentemente letal, particularmente os sarcomas dos tecidos moles que constituem a maioria dos sarcomas diagnosticados (Burningham et al., 2012). No entanto, os dados epidemiológicos e etiológicos traduzem pouco conhecimento científico e de consenso, relativamente aos mecanismos biológicos e fatores de risco ambientais associados a esta doença oncológica (Ibidem).Os sarcomas acarretam um conjunto de sequelas, com consequências negativas ao nível da funcionalidade e autoimagem do doente (Granda-Cameron, Hanlon, Lynch \& Houldin, 2011). As modalidades terapêuticas mais comuns são a cirurgia que pode ser complementada com quimioterapia e/ou radioterapia, as quais também afetam negativamente a funcionalidade e a qualidade de vida (Tang, Pan, Castle \& Choong, 2012) deixando os doentes com sarcoma em situações de dependência e fragilidade emocional.

Os sintomas de ansiedade e de depressão são frequentes na doença oncológica em geral e também nos sarcomas (Ostacoli et al., 2014; Paredes, Canavarro \& Simões, 2011; Paredes, Pereira, Simões \& Canavarro, 2012), podendo atingir níveis de perturbação emocional, a qual interfere na recuperação e qualidade de vida do doente, de tal forma, que o distress emocional foi considerado o sexto sinal vital a avaliar nos doentes com cancro (Bultz et al., 2011). Segundo o National Comprehensive Cancer Network (NCCN) dos Estados Unidos da América, aproximadamente um terço dos doentes com cancro experimenta perturbação emocional, e destes, apenas cerca de $5 \%$ recebe suporte psicológico (NCCN, 2016). O despiste de perturbação emocional e respetivo tratamento, em diferentes fases da doença, tem repercussões positivas no tratamento do doente com cancro (Pirl et al., 2014) e as intervenções psicossociais podem efetivamente reduzir o sofrimento psicológico causado ou agravado pela doença oncológica (Jacobsen \& Jim, 2008; Spears, 2008).

Estão identificados na literatura momentos de maior vulnerabilidade para o desenvolvimento de perturbação emocional no doente com cancro, como por exemplo, o momento do diagnóstico, o período de espera do tratamento, a transição da modalidade de tratamento, o aparecimento de sintomas suspeitos e o fim de tratamento, entre outros (Pirl et al., 2014).
Segundo o NCCN (2016), a avaliação de perturbação emocional deve realizar-se na consulta clínica inicial e repetir-se em intervalos adequados em função da condição clínica do doente e das consultas previstas. Em investigação prévia, fatores demográficos como a idade, o sexo, a raça, o nível educacional e o estado civil, são analisados enquanto possíveis preditores da adaptação emocional do doente com cancro (GrandaCameron et al., 2011; Paredes et al., 2012).

Um outro fator que tem vindo a ser estudado como determinante do ajustamento dos doentes com doença crónica, tem sido o suporte proporcionado pelos profissionais de saúde que acompanham o doente no processo de gestão da doença (Lorig et al. 2016). Neste suporte incluem-se questões como, o fornecimento de informação sobre a doença e o plano terapêutico adaptado às necessidades individuais, o ensino de competências de tomada de decisão e de resolução de problemas, o apoio emocional e o reconhecimento empático de emoções. Mais recentemente a prática clínica tem sido idealmente integrada na filosofia do empowerment, a qual preconiza que os doentes deverão assumir maior controlo sobre a sua saúde e condição clínica, e enquadrada neste paradigma surge a Teoria da Autodeterminação (TAD). A TAD é uma teoria geral do comportamento e motivação humana, a qual defende que o ser humano possui três necessidades psicológicas básicas, ou seja, a autonomia, a competência e o relacionamento, cuja satisfação cria as condições para o crescimento psicológico, integridade e bem-estar (Deci \& Ryan, 2000).

A autonomia reflete a necessidade da pessoa sentir que o seu comportamento resulta da sua vontade e iniciativa. De acordo com Deci e Ryan (2000), a pessoa age de forma autónoma quando se identifica com aquilo que faz, e mesmo quando influenciada por fatores externos, mantém o sentimento de iniciativa e valor. A competência refere-se à necessidade de sentir-se capaz de atingir os resultados desejados, sentindo confiança na sua ação. $O$ relacionamento envolve sentimentos de pertença. A pessoa sente necessidade de estabelecer vínculo com os outros e ser compreendida por eles (Patrick \& Williams, 2012).

O apoio que a pessoa encontra nos outros para satisfazer as suas necessidades básicas, é preditivo de maior autonomia na adoção de comportamentos de saúde (Patrick \& Williams, 2012). Surge assim, o conceito de suporte autónomo, o qual descreve o tipo de suporte proporcionado por alguém (inclusive um enfermeiro) que é sensível ao outro e que não só conhece, mas que também respeita a sua perspetiva, proporcionando escolhas, encorajando a tomada de decisão e a iniciativa (Deci, La Guardia, Moller, Scheinar \& Ryan, 2006). 
Há evidência de que, quando a pessoa sente que é apoiada para satisfazer as necessidades referidas atrás, em determinada situação ou contexto da sua vida, tornase capaz de agir de forma mais autónoma para assumir comportamentos positivos. Por exemplo, a adoção de um comportamento de saúde (Milne, Wallman, Guilfoyle, Gordon \& Corneya, 2008), a adesão terapêutica num contexto de doença crónica (Lemos e Garrett, 2013), ou a melhoria da qualidade de vida numa situação de doença oncológica (Hawkins et al., 2010). Partindo da revisão meta-analítica de vários estudos baseados na TAD, $\mathrm{Ng}$ et al. (2008) reforçam a associação entre a promoção da autonomia e os indicadores de saúde física e de saúde mental (e. g., diminuição da ansiedade e depressão, e melhoria da qualidade de vida). O presente estudo descritivo, correlacional e longitudinal teve como finalidade estudar o ajustamento emocional dos doentes com sarcoma, ao longo do processo de tratamento (no início do tratamento [M1], quatro [M2] e nove meses [M3]) após o início do tratamento), e a sua relação com a perceção sobre o ambiente terapêutico (i. e., suporte autónomo) em dois momentos do processo de tratamento (quatro [M2] e nove meses [M3] após o início do tratamento), considerando também as variáveis sociodemográficas e clínicas. Foram objetivos específicos do estudo: (1) avaliar o ajustamento emocional (sintomas de ansiedade e de depressão), em três momentos do processo de tratamento e a sua variação ao longo do tempo; (2) avaliar a perceção dos doentes sobre o suporte proporcionado pelos enfermeiros, em dois momentos do tratamento e a sua variação ao longo do tempo; (3) descrever a relação entre a ansiedade, a depressão e a perceção de suporte; (4) descrever a relação entre as variáveis sociodemográficas e clínicas, e a perceção de suporte, a ansiedade e a depressão, nos três momentos do tratamento.

\section{METODOLOGIA}

\section{Amostra}

A amostra de conveniência foi constituída por doentes com o diagnóstico de sarcoma (ósseo ou dos tecidos moles), em tratamento em duas instituições de saúde de Portugal Continental.

Os critérios para inclusão na amostra foram: idade superior a 18 anos; com o diagnóstico médico de sarcoma primário dos tecidos moles e/ou ósseo, sem comprometimento cognitivo e que aceitassem livremente participar no estudo.
A amostra foi composta por 24 pessoas com sarcoma, das quais 14 (58\%) eram homens e 10 (42\%) mulheres. Tratou-se de um grupo constituído essencialmente por jovens adultos, com uma média de idades de 41 anos $(\mathrm{DP}=16,8)$, compreendidas entre um mínimo de 18 e um máximo de 66 anos de idade. Metade dos doentes apresentava idade inferior a 40 anos e apenas cinco doentes (21\%) apresentavam mais de 60 anos de idade. A síntese dos dados sociodemográficos e clínicos são apresentados na Tabela 1.

Tabela 1 - Dados Sociodemográficos e Clínicos da Amostra

\begin{tabular}{|c|c|c|c|}
\hline \multicolumn{2}{|l|}{ Variáveis } & $\mathrm{n}$ & $\%$ \\
\hline \multirow{2}{*}{ Sexo } & Feminino & 10 & 41,7 \\
\hline & Masculino & 14 & 58,3 \\
\hline \multirow{3}{*}{ Estado civil } & Casado & 12 & 50 \\
\hline & Solteiro & 11 & 45,8 \\
\hline & Divorciados/separados & 1 & 4,2 \\
\hline \multirow{2}{*}{ Filhos } & Sem filhos & 12 & 50 \\
\hline & Com filhos & 12 & 50 \\
\hline \multirow{2}{*}{ Coabitação } & Família & 23 & 95,8 \\
\hline & Sozinho & 1 & 4,2 \\
\hline \multirow{4}{*}{$\begin{array}{l}\text { Situação pro- } \\
\text { fissional }\end{array}$} & Empregado & 12 & 50 \\
\hline & Desempregado & 1 & 4,2 \\
\hline & Reformado & 5 & 20,8 \\
\hline & Estudante & 5 & 20,8 \\
\hline \multirow{3}{*}{$\begin{array}{l}\text { Tipo de } \\
\text { tumor }\end{array}$} & Outro & 1 & 4,2 \\
\hline & Tecidos moles & 15 & 62,5 \\
\hline & Ósseo & 9 & 37,5 \\
\hline
\end{tabular}

Apresentavam ainda uma média de 10 anos de escolaridade $(\mathrm{DP}=4,3)$ e em termos profissionais, metade da amostra encontrava-se empregada, mas na sua maioria com baixa clínica $(n=9 ; 37,5 \%)$ e apenas um doente estava desempregado. Relativamente à profissão e, de acordo com a Classificação Nacional de Profissões, seis doentes enquadravam-se na categoria de pessoal dos serviços e vendedores, seis doentes pertenciam à categoria de quadros superiores ou profissões intelectuais e científicas, quatro doentes aos operários, artífices e similares, um doente pertencia ao grupo do pessoal administrativo e similares, e seis doentes eram domésticas ou estudantes (categoria criada para este estudo). Em termos clínicos, a maioria dos doentes apresentava tumores das partes moles $(n=15 ; 62,5 \%)$, e em relação às modalidades terapêuticas, a maioria dos doentes foi submetida a cirurgia $(n=17 ; 70,8 \%)$, à qual foram associadas a quimioterapia e/ou a radioterapia. Os restantes doentes foram submetidos apenas a quimioterapia $(n=7 ; 29,2 \%)$. 
As tipologias cirúrgicas realizadas foram díspares, desde as exéreses de tumores/sarcomas, a exéreses de metástases ou plastias cutâneas. Nenhum dos doentes foi submetido a amputação.

\section{Instrumentos}

\section{Questionário sociodemográfico e clínico.}

Questionário composto por questões de resposta curta, criado para o presente estudo. As variáveis avaliadas foram: sexo, idade, escolaridade, estado civil, número de filhos, coabitação e tipo de tumor.

\section{Ansiedade e depressão.}

A avaliação dos sintomas de ansiedade e de depressão foi realizada com a versão portuguesa da Escala de Ansiedade e Depressão Hospitalar (EADH) de Pais-Ribeiro et al. (2007). Esta escala é um instrumento de autorresposta que permite identificar os componentes emocionais associados à doença física, sendo composto por duas subescalas cotadas separadamente: uma que avalia a ansiedade (sete itens) e outra que avalia a depressão (sete itens). Cada item está formulado para uma escala de resposta de tipo Likert com quatro pontos (0-3), pelo que os valores possíveis para cada uma das subescalas variam entre zero e 21. Os pontos de cut-off escolhidos foram baseados no estudo de validação da versão portuguesa, em que resultados compreendidos entre zero e sete devem ser interpretados como "normal", entre oito e 10 "ligeira", entre 11 e 14 "moderada" e entre 15 e 21 "grave", quer para a subescala ansiedade, quer para a subescala depressão (Pais-Ribeiro et al., 2007). Relativamente ao resultado global da $\mathrm{EADH}$, valores iguais ou superiores a 15 são considerados como sugestivos de níveis patológicos. A fidelidade foi analisada na amostra em estudo (M1), tendo-se obtido os seguintes valores de alfa de cronbach: EADH - 0,84; Subescala ansiedade - 0,82; Subescala depressão - 0,65.

\section{Suporte autónomo.}

A versão portuguesa do Questionário da Perceção do Cliente sobre o Ambiente Terapêutico (QPCAT) adaptado por Lemos e Garrett (2013) foi utilizada para recolher os dados sobre a perceção dos doentes em relação ao suporte proporcionado pelos enfermeiros. Este instrumento é constituído por seis itens, pontuados numa escala tipo Likert de um (afirmação nada verdadeira) a sete (afirmação totalmente verdadeira). O resultado obtém-se através da soma da pontuação obtida em cada um dos seis itens (a variar entre seis e 42).
Quanto mais elevado o valor total, maior é o nível de suporte proporcionado pelos enfermeiros e que é incentivador da autonomia do doente. $\mathrm{O}$ valor de alfa de cronbach na amostra em estudo foi de 0,91 (M2).

\section{Procedimento}

Os doentes foram contactados no momento da primeira consulta de início de tratamento, altura em que foi realizado o pedido para participação no estudo e a primeira recolha de dados (M1).

Estes mesmos doentes aceitaram ainda ser posteriormente contactados para responderem ao questionário, sendo que a entrevista decorria no dia em que os doentes se deslocavam ao hospital para uma consulta integrada no protocolo terapêutico. A recolha de dados relativos à perceção sobre o ambiente terapêutico (QPCAT) foi realizada apenas no segundo [M2] e terceiro momentos de contacto [M3], considerando que no primeiro momento [M1] era improvável a existência de contacto prévio com os enfermeiros.

A recolha de dados foi realizada por enfermeiros das referidas instituições, colaboradores no presente estudo e previamente preparados para o efeito. Todos os procedimentos do estudo foram realizados de acordo com a melhor prática clínica de cada instituição.

Para a implementação deste estudo foram cumpridos todos os pressupostos éticos inerentes à investigação em saúde. Para além da assinatura do consentimento informado para a participação na investigação, foi ainda obtida autorização das instituições e respetivas comissões de ética, bem como dos autores dos instrumentos utilizados.

\section{Análise dos Dados}

Os dados foram analisados com recurso à estatística descritiva e inferencial, utilizando o programa SPSS (Statistics Package for the Social Sciences), versão 21. $\mathrm{Na}$ análise exploratória da distribuição dos dados percebemos que apenas algumas variáveis apresentavam distribuição normal, e considerando ainda o tamanho da amostra, optámos por utilizar a estatística não paramétrica.

\section{RESULTADOS}

No sentido de avaliar o ajustamento emocional (sintomas de ansiedade e de depressão), em três momentos do processo de doença e a sua variação ao longo do tempo, foi feita uma análise descritiva dos resultados obtidos com a EADH (Tabela 2). 
Tabela 2 - Análise Descritiva dos Sintomas de Ansiedade e Depressão, de acordo com os Pontos de Corte da EADH

\begin{tabular}{|l|c|c|c|c|c|c|}
\hline \multirow{2}{*}{$\begin{array}{l}\text { Nível de } \\
\text { sintomas }\end{array}$} & \multicolumn{3}{|c|}{ Ansiedade } & \multicolumn{3}{c|}{ Depressão } \\
\cline { 2 - 7 } & $\begin{array}{c}\text { M1 } \\
\mathrm{n}(\%)\end{array}$ & $\begin{array}{c}\mathrm{M} 2 \\
\mathrm{n}(\%)\end{array}$ & $\begin{array}{c}\mathrm{M} 3 \\
\mathrm{n}(\%)\end{array}$ & $\begin{array}{c}\text { M1 } \\
\mathrm{n}(\%)\end{array}$ & $\begin{array}{c}\text { M2 } \\
\mathrm{n}(\%)\end{array}$ & $\begin{array}{c}\text { M3 } \\
\mathrm{n}(\%)\end{array}$ \\
\hline Normal & $\begin{array}{c}12 \\
(50)\end{array}$ & $\begin{array}{c}13 \\
(54,2)\end{array}$ & $\begin{array}{c}15 \\
(62,5)\end{array}$ & $\begin{array}{c}18 \\
(75)\end{array}$ & $\begin{array}{c}18 \\
(75)\end{array}$ & $\begin{array}{c}18 \\
(75)\end{array}$ \\
\hline Ligeiro & 8 & 7 & 5 & 6 & 3 & 5 \\
$(33,3)$ & $(29,2)$ & $(20,8)$ & $(25)$ & $(12,5)$ & $(20,8)$ \\
\hline $\begin{array}{l}\text { Mod- } \\
\text { erado }\end{array}$ & $\begin{array}{c}4 \\
(16,7)\end{array}$ & $\begin{array}{c}3 \\
(12,5)\end{array}$ & $\begin{array}{c}4 \\
(16,7)\end{array}$ & 0 & 2 & 0 \\
\hline Grave & 0 & 0 & 0 & 0 & 0 & 0 \\
\hline
\end{tabular}

Os resultados da EADH permitem-nos perceber que grande parte dos participantes ( $\geq 50 \%$ ), não apresentava níveis clínicos de ansiedade, tendo aumentado esse número ao longo dos três momentos de avaliação. No entanto, alguns participantes apresentavam ansiedade ligeira ou até moderada. Nenhum participante apresentou ansiedade de nível grave. Em relação aos sintomas de depressão, uma grande maioria dos participantes (75\%) não apresentou sintomas clínicos de depressão, nos três momentos de avaliação. No entanto, podemos perceber que alguns participantes apresentaram níveis clínicos de depressão, embora ligeiros, nos três momentos de avaliação, com dois participantes a apresentarem níveis moderados no segundo momento de avaliação. Nenhum participante apresentou depressão de nível grave. A comparação entre as médias, nas escalas de avaliação da ansiedade e da depressão, realizada com o recurso ao teste de Friedman, não demonstrou diferenças estatisticamente significativas entre os três momentos avaliados.

No sentido de avaliar a perceção dos doentes sobre o suporte proporcionado pelos enfermeiros, em dois momentos do tratamento (M2 e M3), assim como a sua variação ao longo do tempo, realizou-se a sua análise descritiva, bem como um teste de comparação de médias, cujos resultados são apresentados na tabela seguinte (Tabela 3).

Tabela 3 - Análise Descritiva e Comparação de Médias do QPCAT

\begin{tabular}{|c|c|c|c|}
\hline \multirow{3}{*}{ QPCAT } & $\begin{array}{c}\text { M2 } \\
\text { M (DP) }\end{array}$ & $\begin{array}{c}\text { M3 } \\
\text { M (DP) }\end{array}$ & $\begin{array}{c}\text { Teste de Wilcoxon } \\
(\mathrm{Z} ; \mathrm{p})\end{array}$ \\
\cline { 2 - 5 } & $36,7(4,86)$ & $36,0(5,63)$ & $\mathrm{Z}=-0,44 ; \mathrm{ns}$ \\
\hline
\end{tabular}

Em relação à perceção acerca do ambiente terapêutico, a análise dos resultados permite-nos verificar que nos dois momentos, a perceção acerca do suporte autónomo proporcionado pelos enfermeiros se encontra bastante acima do ponto médio da escala, o que nos permite inferir que os doentes tinham uma perceção positiva sobre a relação estabelecida com os enfermeiros, em ambos os momentos avaliados.

Com base nos resultados obtidos através do teste de Wilcoxon, podemos verificar a inexistência de uma diferença significativa entre os scores obtidos nos dois momentos de avaliação, sugerindo que os enfermeiros constituíram uma fonte de suporte promotor da autonomia dos doentes ao longo do processo de tratamento. No sentido de compreendermos a relação existente entre a ansiedade, a depressão e a perceção de suporte, realizámos a uma análise da correlação, com $\mathrm{R}$ de Spearman, entre os resultados obtidos no QPCAT e as subescalas de ansiedade e depressão da $\mathrm{EADH}$ nos dois momentos do tratamento (M2 e M3), os quais são apresentados na Tabela 4.

Tabela 4 - Correlação R de Spearman entre o QPCAT e as Subescalas da EADH

\begin{tabular}{|c|l|c|c|c|c|}
\hline \multirow{4}{*}{ QPCAT } & \multicolumn{4}{|c|}{ EADH } \\
\cline { 3 - 6 } & & \multicolumn{2}{|c|}{ Ansiedade } & \multicolumn{2}{c|}{ Depressão } \\
\cline { 3 - 6 } & & $\mathrm{M} 2$ & $\mathrm{M} 3$ & $\mathrm{M} 2$ & $\mathrm{M} 3$ \\
\cline { 2 - 6 } & M1 & $\mathrm{ns}$ & $-0,42^{\star}$ & $\mathrm{ns}$ & $-0,41^{\star}$ \\
\cline { 2 - 6 } & $\mathrm{M} 2$ & $\mathrm{n}$ & $-0,61^{* *}$ & $\mathrm{~ns}$ & $-0,51^{\star}$ \\
\hline
\end{tabular}

${ }^{*} \mathrm{p}<0,05 ;{ }^{* *} \mathrm{p}<0,01$

Como se pode verificar pelos valores da tabela anterior, os sintomas de ansiedade e de depressão apresentaram uma associação negativa e significativa com o suporte proporcionado pelos enfermeiros, apenas no terceiro momento de avaliação, o que nos permite inferir que, na fase mais avançada do tratamento, os doentes que percecionaram mais suporte autónomo por parte destes profissionais, também apresentaram menores sintomas de ansiedade e de depressão.

No sentido de dar resposta ao último objetivo deste estudo, foi testada a relação entre as variáveis sociodemográficas e clínicas, com a perceção de suporte, a ansiedade e a depressão, da qual salientamos os resultados mais significativos:

- A idade das doentes com sarcoma apresentou uma correlação moderada, positiva e significativa com o score relativo à depressão, nos três momentos de avaliação: (M1: Rs = 0,53; $\mathrm{p}<0,01) ; \mathrm{M} 2$ : Rs = 0,50; $\mathrm{p}<0,05$ e M3: $\mathrm{Rs}=0,44 ; \mathrm{p}<0,05)$, indicando que os doentes mais velhos relataram mais sintomas do tipo depressivo durante todo o processo de tratamento.

- O estado civil mostrou ser uma variável associada aos sintomas de ansiedade no início do tratamento (M1), 
tendo os doentes com parceiro (casados ou em união de facto) ( $\mathrm{n}=12$; $\mathrm{MR}=16,8)$ apresentado valores superiores de ansiedade, comparativamente com os doentes solteiros ( $\mathrm{n}=11 ; \mathrm{MR}=8,6)$ e divorciados/separados ( $\mathrm{n}$ $=1 ; \mathrm{MR}=4)$, com X2 (2) = 9,22; p =0,01. Também relativamente aos sintomas depressivos, os doentes com parceiro $(\mathrm{n}=12$; $\mathrm{MR}=15,3)$ apresentaram valores superiores de depressão, comparativamente com os doentes solteiros ( $\mathrm{n}=10 ; \mathrm{MR}=8,2)$ e divorciados/separados ( $\mathrm{n}=1 ; \mathrm{MR}=10,5)$, no terceiro momento de avaliação, $\operatorname{com~X} 2(2)=6,27 ; p=0,04$.

- O número de filhos das pessoas que integraram a amostra, apresentou também uma correlação positiva, moderada e significativa com a ansiedade, no início do tratamento $(\mathrm{M} 1)(\mathrm{Rs}=0,43 ; \mathrm{p}<0,05)$, indicando que as pessoas com mais filhos relataram mais sintomas de ansiedade.

- Por outro lado, o número de pessoas que coabitavam com o doente mostrou apresentar uma correlação moderada e negativa com a presença de sintomas depressivos no primeiro momento de avaliação com Rs = - 0,44; $\mathrm{p}<0,05$, parecendo significar que quanto maior era $\mathrm{o}$ número de conviventes familiares, menor era a presença de sintomas depressivos.

- Também a situação profissional (ativo ou não ativo) se mostrou associada à presença de sintomas depressivos no segundo momento de avaliação $(Z=-2,42 ; p=0,01)$, uma vez que as médias dos doentes que se encontravam no ativo $(\mathrm{n}=3$; $M R=2,17)$ apresentaram-se significativamente inferiores aos doentes que se encontravam com baixa clínica $(\mathrm{n}=9 ; \mathrm{MR}=7,94)$. $\mathrm{Na}$ análise das restantes variáveis sociodemográficas (sexo e escolaridade) e das variáveis clínicas (tipo de tumor e tempo após diagnóstico) não foram encontradas diferenças estatisticamente significativas entre os grupos.

\section{DISCUSSÃO}

Este estudo teve como finalidade estudar a relação entre a perceção sobre o ambiente terapêutico, mais especificamente o suporte autónomo proporcionado pelos enfermeiros, e o ajustamento emocional nos doentes com sarcoma, ao longo da trajetória de tratamento da doença. Os resultados descritivos apontam, globalmente, para a presença de alguns sintomas de ansiedade e de depressão ao longo do tratamento, bem como, uma perceção positiva do suporte proporcionado pelos enfermeiros.

$\mathrm{Na}$ literatura, vários estudos identificam a presença de ansiedade e de depressão nos doentes com sarcoma em diferentes fases da doença (Granda-Cameron et al.,
2011; Ostacoli et al., 2014; Paredes et al., 2011; Paredes et al., 2012;), assim como a importância do suporte prestado pelos profissionais de saúde aos doentes com cancro, na promoção da sua autonomia (Milne et al., 2008). De realçar que, no presente estudo, os doentes não apresentaram níveis graves de ansiedade e de depressão, nos três momentos de avaliação, o que vai ao encontro dos resultados de outros estudos, em que a maioria dos doentes com sarcoma não experimentam problemas significativos no seu ajustamento emocional, existindo apenas uma pequena percentagem de doentes com níveis clinicamente significativos e graves de sintomatologia (Ostacoli et al., 2014; Paredes et al., 2012). Em concordância com a análise de outros investigadores, parece-nos que os resultados do presente estudo mostram que, apesar do sofrimento vivenciado, os doentes conseguem lidar com a doença de forma adaptativa. Convém, no entanto, realçar, que os sintomas de ansiedade e de depressão, dentro de níveis ligeiros ou moderados, constituem reações transitórias frequentes e normativas no processo de ajustamento, em parte fruto da exigência física e psicológica das modalidades terapêuticas (Granda-Cameron et al., 2011; Paredes et al., 2011). Apesar do seu número reduzido, estas pessoas que apresentam sintomas ligeiros a moderados devem ser sinalizados e necessitam da atenção e da intervenção por parte dos profissionais de saúde. Na verdade, a avaliação do impacto emocional é imperativa na fase inicial de diagnóstico e ao longo da trajetória da doença, de forma a possibilitar a ajuda psicológica aos doentes com perturbação emocional (NCCN, 2016; Paredes et al., 2012; Pirl et al., 2014).

De acordo com a avaliação dos nossos participantes, podemos afirmar que os enfermeiros proporcionaram aos doentes opções para a resolução de problemas associados à sua doença, bem como, compreenderam e demonstraram confiança na sua capacidade de seguir o tratamento indicado, encorajando-os a colocarem questões. Estes resultados evidenciam que os enfermeiros adotaram, predominantemente, um suporte do tipo autónomo, o que segundo os pressupostos da TAD, é promotor de resultados positivos no domínio da saúde (Deci et al., 2006; Hawkins et al., 2010; Lemos e Garrett, 2013; Milne et al., 2008; Patrick \& Williams, 2012).

No que diz respeito ao domínio mais específico dos sarcomas, não encontramos estudos sustentados na TAD, relativamente ao ambiente terapêutico e ao tipo de suporte proporcionado pelos profissionais a estes doentes, pelo que, os nossos resultados não podem ser comparados com precisão. 
Porém, globalmente, a literatura aponta para uma associação do suporte percecionado pelo doente oncológico com um melhor ajustamento psicológico ao longo do processo de tratamento. Por exemplo, no estudo de Spears (2008) os doentes com sarcoma reconhecem a importância da intervenção das enfermeiras em resposta à sua necessidade de suporte emocional.

Um resultado importante do nosso estudo é que a perceção globalmente positiva do suporte proporcionado pelos enfermeiros se associa à diminuição de sintomas de ansiedade e de depressão, nomeadamente nas fases avançadas do tratamento. Estes resultados vão ao encontro das conclusões de uma meta-análise realizada com 184 estudos, em que o desenvolvimento da autonomia, dos doentes com diversos quadros patológicos, foi promotora de uma melhor saúde mental ( $\mathrm{Ng}$ et al., 2008). Outros estudos descrevem, de forma mais específica, os benefícios do suporte autónomo, por exemplo, num estudo de Deci et al. (2006), o suporte proporcionado por amigos chegados foi identificado como determinante do bem-estar e saúde psicológica da população geral. Por sua vez, Milne et al. (2008) demonstraram a importância do suporte autónomo proporcionado por profissionais de saúde na promoção da atividade física, em sobreviventes de cancro da mama. Por fim, no estudo de Hawkins et al. (2010), a utilização de uma intervenção promotora da autonomia, competência e relacionamento, num grupo de mulheres com cancro da mama, levou à melhoria da sua qualidade de vida, comparativamente com um grupo de controlo que não recebeu esta intervenção, sendo a intervenção considerada uma variável mediadora.

Curiosamente, no nosso estudo, a associação entre o suporte autónomo e a diminuição dos sintomas de ansiedade e de depressão, fez-se sentir no último momento de avaliação (M3), por isso numa fase mais avançada do tratamento. Este resultado pode, eventualmente, relacionar-se com o estabelecimento de uma relação de maior confiança com os profissionais de saúde, fruto dos contactos em maior número, os quais possibilitaram ao doente mais oportunidades de receber informação e suporte.

O que não nos surpreende, uma vez que, com o passar do tempo, o doente tende a adaptar-se à sua condição de saúde e às exigências físicas e psicológicas dos tratamentos, particularmente quando recebe apoio (Jacobsen \& Jim, 2008; Pirl et al., 2014). No entanto, há estudos que documentam a ausência deste processo adaptativo ao longo do tempo, relatando, em contrapartida, um aumento progressivo dos sintomas de ansiedade e de depressão ao longo das fases de tratamento (GrandaCameron et al., 2011).
Os resultados do presente estudo indicaram ainda que os doentes de mais idade, com parceiro (casados ou em união de facto), com um número reduzido de conviventes na habitação e em situação de baixa clínica, apresentaram maiores níveis de depressão, em diferentes momentos do tratamento. Por outro lado, os doentes com parceiro (casados ou em união de facto) e com um maior número de filhos, apresentaram níveis mais elevados de ansiedade, em algum momento do tratamento. Estes resultados permitem-nos identificar os doentes que apresentam maior vulnerabilidade, necessitando de ser acompanhados e apoiados nas diferentes fases da doença. No nosso estudo, a idade é uma variável que se associa a maiores níveis de depressão ao longo do tratamento, um resultado semelhante é referido por Granda-Cameron et al. (2011), em que os participantes mais velhos apresentavam, na sua generalidade, um aumento de sintomas de perturbação física e psicológica ao longo dos ciclos de tratamento por quimioterapia, enquanto os participantes mais novos se mantinham mais estáveis na expressão destes sintomas.

Contrariamente às nossas expectativas, constatámos que o estar casado ou viver em união de facto, se associa a níveis mais elevados de ansiedade e à presença de sintomas depressivos ao longo do processo de tratamento. Este resultado foi também referido num outro estudo em Portugal, realizado com doentes com sarcoma (Paredes et al., 2012) e pode encontrar explicação no facto de os doentes terem de lidar não só com o seu sofrimento, mas também com o sofrimento psicológico dos seus parceiros. Os resultados revelam que o impacto emocional da doença nos parceiros é uma variável importante a considerar nas intervenções a estes doentes e suas famílias. Os profissionais de saúde podem ajudar os casais a lidar com a mudança imposta pela doença e reequacionar os seus papéis pessoais e sociais, particularmente durante a fase de tratamento (Paredes et al., 2012).

Uma variável que estudamos e pode ajudar a compreender, de uma forma ainda mais detalhada, as relações familiares e seu impacto na adaptação à doença, foi o número de filhos e de coabitantes. Neste estudo, os doentes com um maior número de filhos apresentaram mais sintomas depressivos, na fase inicial do tratamento. Também neste caso, os doentes acumulam as exigências da doença e do tratamento, com a sua função parental e a necessidade de manterem o cuidado aos filhos, criando, eventualmente, expectativas negativas, ancoradas na incerteza face a um futuro desconhecido. 
O que já não se verifica na fase final do tratamento, provavelmente porque os doentes ao lidarem com a realidade do fim das terapêuticas, adquirem sentimentos de esperança e de maior controlo sobre as situações que lhes dizem respeito, o que facilita a adaptação à doença, amenizado assim as expectativas mais negativas. Em contrapartida, coabitar com maior número de pessoas parece favorecer o processo de ajustamento à doença e seu tratamento. Não encontramos estudos que analisassem estas variáveis e, por conseguinte, não nos foi possível, mais uma vez, comparar os resultados encontrados.

Finalmente, os nossos resultados também evidenciam a situação profissional como uma variável que se associa à sintomatologia depressiva. Os doentes que se encontram em baixa clínica exibem mais sintomas depressivos do que os doentes que se encontram no ativo, o que poderá indicar que a ausência de atividade pode ser percursora da presença de um maior número de sintomas depressivos. No entanto, esta associação verifica-se apenas no primeiro momento de avaliação (M1), e mais uma vez a incerteza do desconhecido e a falta de controlo, pode estar na explicação deste resultado. Acresce que os doentes com baixa clínica, provavelmente, também estão mais fragilizados e vulneráveis, nomeadamente em termos físicos, razão porque o médico atribui o atestado de doença, justificando a sua incapacidade temporária para exercer a sua atividade laboral. Em contrapartida, os doentes em situação de baixa clinica demonstraram uma melhor perceção sobre o apoio proporcionado pelos enfermeiros.

Não encontrámos outras associações entre as variáveis sociodemográficas e clínicas e o ajustamento emocional dos doentes com sarcoma, tal como aconteceu com outros estudos (Paredes et al., 2012). Como já referimos anteriormente, não existe investigação prévia sobre o papel do suporte autónomo no ajustamento emocional dos doentes com sarcoma, por conseguinte, o nosso estudo parece-nos ser pioneiro ao procurar aplicar os princípios da TAD ao estudo das vivências do doente com sarcoma.

Foi fator de limitação do nosso estudo o número reduzido de participantes, todavia, sendo o sarcoma um cancro raro, é natural que o universo de doentes seja parco. A juntar a este facto acresce que, no nosso país, existem várias instituições de referência vocacionadas para o acompanhamento destes doentes, o que leva à sua dispersão, fator que também dificultou o acesso aos potenciais participantes.

\section{CONCLUSÕES}

O estudo desenvolvido sobre o ajustamento emocional dos doentes com sarcoma em duas instituições de saúde portuguesas revelou níveis baixos de sintomatologia ansiosa e depressiva, com uma ligeira melhoria dos sintomas de ansiedade ao longo da trajetória da doença e seu tratamento. Os doentes demonstraram também uma perceção bastante positiva sobre o suporte proporcionado pelos enfermeiros, com repercussões positivas no seu ajustamento emocional, em especial nas fases mais avançadas do processo de tratamento. Foi possível ainda perceber que algumas variáveis sociodemográficas, como a idade, o estado civil, o número de filhos e de pessoas que coabitam com o doente, e ainda a sua situação profissional (estar ativo ou com baixa clínica), influenciavam significativamente a vivência da doença. Estes resultados sugerem que os enfermeiros proporcionam aos doentes um suporte emocional de tipo autónomo, apresentando opções para a resolução dos problemas associados à sua doença, compreendem e demonstram confiança na sua capacidade para gerir a doença e o seu tratamento, encorajando-os a colocarem dúvidas e procurarem ajuda. De acordo com os pressupostos da TAD, este suporte é promotor de resultados positivos no domínio da saúde/doença. Os resultados identificam ainda um conjunto de indicadores (sociodemográficos) de maior vulnerabilidade à presença de níveis clínicos de perturbação emocional e que requerem um suporte terapêutico continuado ao longo de todo o percurso da doença.

\section{REFERÊNCIAS BIBLIOGRÁFICAS}

Bultz, B. D., Groff, S. L., Fitch, M., Blais, M. C., Howes, J., Levy, K., \& Mayer, C. (2011). Implementing screening for distress, the 6th vital sign: a Canadian strategy for changing practice. Psycho-Oncology, 20(5), 463469.

Burningham, Z., HashibE, M., Spector, L., \& Schiffman, J. (2012). The epidemiology of sarcoma. Clinical Sarcoma Research, 2(14). Acedido em http://www.clinicalsarcomaresearch.com/content/2/1/14

Deci, E. L., \& Ryan, R. M. (2000). The 'what' and 'why' of goal pursuits: Human needs and the self-determination of behavior. Psychological Inquiry, 11, 227-268. 
Deci, E. L., La Guardia, J. G., Moller, A. C., Scheiner, M. J., \& Ryan, R. M. (2006). On the benefits of giving as well as receiving autonomy support: Mutuality in close friendships. Personality \& Social Psychology Bulletin, 32(3), 313-327.

Granda-Cameron, C., Hanlon, A. L., Lynch, M. P., \& Houldin, A. (2011). Experience of newly diagnosed patients with sarcoma receiving chemotherapy. Oncology Nursing Forum, 38(2), 160-169. doi: dx.doi. org/10.1188/11.onf.160-169

Hawkins, R. P., Pingree, S., Shaw, B., Serlin, R. C., Swoboda, C., Han, J. Y., Carmack, S. L., \& Salner, A. (2010). Mediating processes of two communication interventions for breast cancer patients. Patient Education \& Counseling, 81, S48-S53. doi: dx.doi.org/10.1016/j. pec.2010.10.021

Jacobsen, P. B., \& Jim, H. S. (2008). Psychosocial interventions for anxiety and depression in adult cancer patients: Achievements and challenges. CA: A Cancer Journal For Clinicians, 58(4), 214-230. doi: dx.doi. org/10.3322/CA.2008.0003

Lemos, M., e Garrett, S. (2013). HCCQ: H-D Questionário de Perceção do Ambiente Terapêutico: Saúde - Diabetes. In M. S. Lemos, A. M. Gamelas, e J. A. Lima (Eds.), Instrumentos de investigação desenvolvidos, adaptados ou usados pelo grupo de investigação desenvolvimental, educacional e clínica com crianças e adolescentes. Porto: Universidade do Porto, Faculdade de Psicologia e de Ciências da Educação.

Lorig, K., Ritter, P. L., Turner, R. M., English, K., Laurent, D. D., \& Greenberg, J. (2016). Benefits of diabetes self-management for health plan members: A 6-month translation study. Journal of Medical Internet Research, 18(6), e164-e174. doi: dx.doi.org/10.2196/jmir.5568

Milne, H. M., Wallman, K. E., Guilfoyle, A., Gordon, S., \& Courneya, K. S. (2008). Self-determination theory and physical activity among breast cancer survivors. Journal of Sport \& Exercise Psychology, 30(1), 23-38.

National Comprehensive Cancer Network. (2016). Managing stress and distress. Acedido em https://www. nccn.org/patients/resources/life_with_cancer/distress. aspx
Ng, J. Y., Ntoumanis, N., Thøgersen-Ntoumani, C., Deci, E. L., Ryan, R. M., Duda, J. L., \& Williams, G. C. (2012). Self-determination theory applied to health contexts: A meta-analysis. Perspectives On Psychological Science, 7(4), 325-340. doi: dx.doi. org/10.1177/1745691612447309

Ostacoli, L., Saini, A., Zuffranieri, M., Boglioni, A., Carletto, S., De Marco, I., Lombardi, I., Picci, R. L., Berruti, A., \& Comandone, A. (2014). Quality of life, anxiety and depression in soft tissue sarcomas as compared to more common tumours: An observational study. Applied Research Quality of Life, 9, 123-131.

Pais-Ribeiro, J., Silva, I., Ferreira, T., Martins, A., Meneses, R., e Baltar, M. (2007). Validation study of a Portuguese version of the Hospital Anxiety and Depression Scale. Psychology Health Medicine, 12(2), 225-235. doi: dx.doi.org/10.1080/13548500500524088

Paredes, T. T., Pereira, M. M., Simões, M. R., e Canavarro, M. C. (2012). A longitudinal study on emotional adjustment of sarcoma patients: The determinant role of demographic, clinical and coping variables. European Journal of Cancer Care, 21(1), 41-51. doi: dx.doi. org/10.1111/j.1365-2354.2011.01269.x

Paredes, T., Canavarro, M. C., e Simões, M. R. (2011). Anxiety and depression in sarcoma patients: Emotional adjustment and its determinants in the different phases of disease. European Journal of Oncology Nursing, 15(1), 73-79. doi: dx.doi.org/10.1016/j.ejon.2010.06.004

Patrick, H., \& Williams, G. C. (2012). Self-determination theory: Its application to health behavior and complementarity with motivational interviewing. The International Journal of Behavioral Nutrition and Physical Activity, 918. doi: dx.doi.org/10.1186/1479-5868-918

Pirl, W. F., Fann, J. R., Greer, J. A., Braun, I., Deshields, T., Fulcher, C., \& ... Bardwell, W. A. (2014). Recommendations for the implementation of distress screening programs in cancer centers: Report from the American Psychosocial Oncology Society, Association of Oncology Social Work, and Oncology Nursing Society joint task force. Cancer, 120(19), 2946-2954. doi: dx.doi. org/10.1002/cncr.28750 
Spears, J. (2008). Emotional support given by wardbased nurses to sarcoma patients. European Journal of Oncology Nursing, 12(4), 334-341. doi: dx.doi. org/10.1016/j.ejon.2008.03.001
Tang, M., Pan, D., Castle, D., \& Choong, P. (2012). A systematic review of the recent quality of life studies in adult extremity sarcoma survivors. Sarcoma, 15. doi: dx.doi.org/10.1155/2012/171342

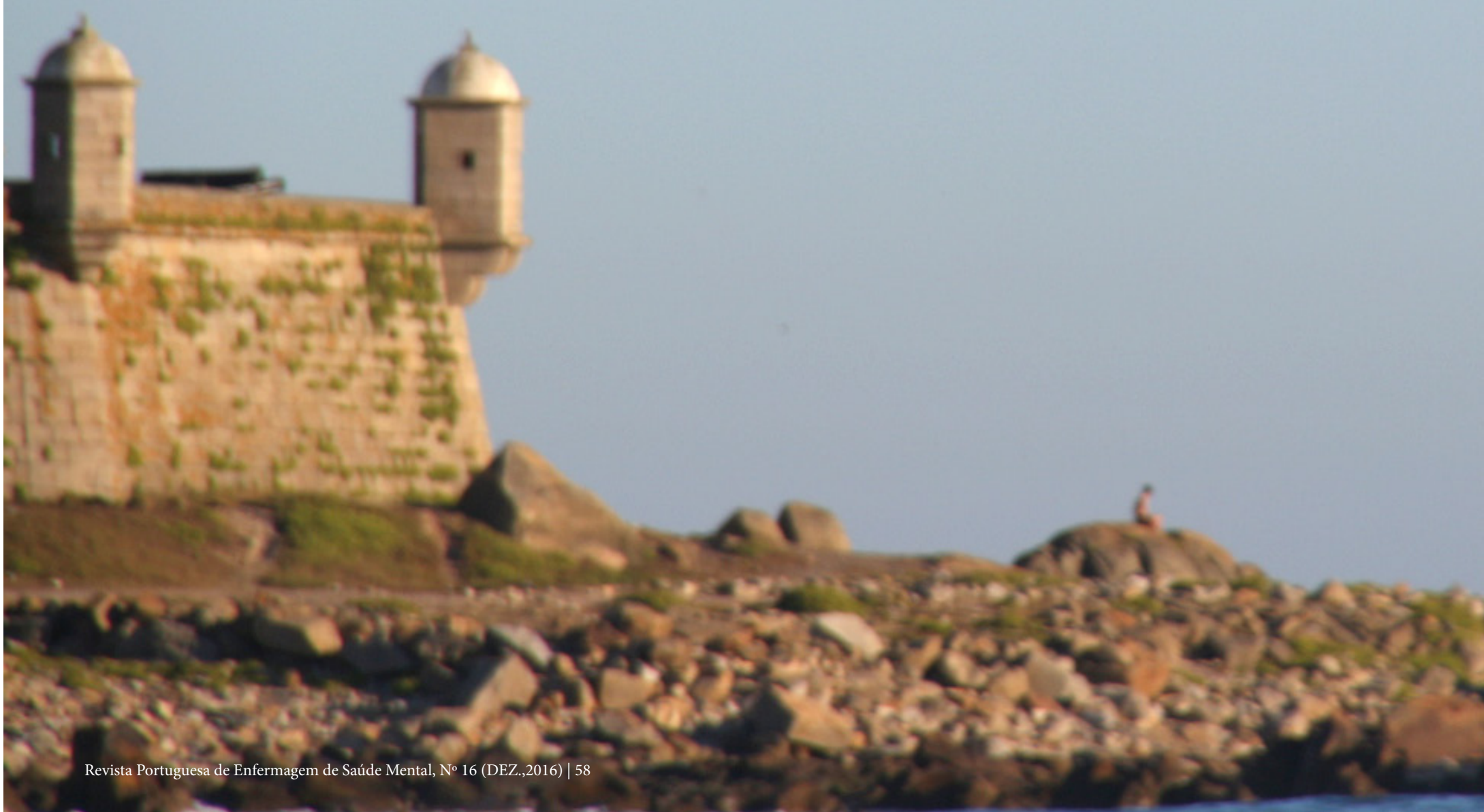

